\title{
A NOTE ON EXISTENCE AND NON-EXISTENCE OF HORIZONS IN SOME ASYMPTOTICALLY FLAT 3-MANIFOLDS
}

\author{
Pengzi Miao
}

\begin{abstract}
We consider asymptotically flat manifolds of the form $\left(S^{3} \backslash\{P\}, G^{4} g\right)$, where $G$ is the Green's function of the conformal Laplacian of $\left(S^{3}, g\right)$ at a point $P$. We show if $\operatorname{Ric}(g) \geq 2 g$ and the volume of $\left(S^{3}, g\right)$ is no less than one half of the volume of the standard unit sphere, then there are no closed minimal surfaces in $\left(S^{3} \backslash\{P\}, G^{4} g\right)$. We also give an example of $\left(S^{3}, g\right)$ where $\operatorname{Ric}(g)>0$ but $\left(S^{3} \backslash\{P\}, G^{4} g\right)$ does have closed minimal surfaces.
\end{abstract}

\section{Introduction}

Let $\left(N^{3}, g, p\right)$ be an initial data set satisfying the dominant energy constraint condition in general relativity. It is a fascinating question to ask under what conditions an apparent horizon (of a back hole) exists in $\left(N^{3}, g, p\right)$. Here an apparent horizon is a 2-surface $\Sigma^{2} \subset N^{3}$ satisfying

$$
H_{\Sigma}=\operatorname{Tr}_{\Sigma} p
$$

where $H_{\Sigma}$ is the mean curvature of $\Sigma$ in $N$ and $\operatorname{Tr}_{\Sigma} p$ is the trace of the restriction of $p$ to $\Sigma$.

A fundamental result of Schoen and Yau states that matter condensation causes apparent horizons to be formed [11]. Their result is remarkable not only because it provides a general criteria to the existence question, but also because it leads to a refined problem - besides matter fields, what is the pure effect of gravity on the formation of apparent horizons?

To analyze this refined problem, one considers an asymptotically flat initial data set $\left(N^{3}, g, p\right)$ in a vacuum spacetime. As the first step, one assumes $\left(N^{3}, g, p\right)$ is timesymmetric (i.e. $p \equiv 0$ ). In this context, an apparent horizon is simply a minimal surface, and the relevant topological assumption is that $N^{3}$ is diffeomorphic to $\mathbb{R}^{3}$. (If $N^{3}$ has nontrivial topology, a closed minimal surface always exists by [8].)

There is a geometric construction of such an initial data set. Let $[g]$ be a conformal class of metrics on the three-sphere $S^{3}$. Recall the Yamabe constant of $\left(S^{3},[g]\right)$ is defined by

$$
Y\left(S^{3},[g]\right)=\inf _{v \in W^{1,2}\left(S^{3}\right)} \frac{\int_{M}\left[8|\nabla v|_{g}^{2}+R(g) v^{2}\right] d V_{g}}{\left(\int_{M} v^{6} d V_{g}\right)^{\frac{1}{3}}}
$$

where $R(g)$ is the scalar curvature of $g$. If $Y\left(S^{3},[g]\right)>0$, there exists a positive Green's function $G$ of the conformal Laplacian $8 \triangle_{g}-R(g)$ at any fixed point $P \in S^{3}$. Consider the new metric $G^{4} g$ on $S^{3} \backslash\{P\}$, it is easily checked that $\left(S^{3} \backslash\{P\}, G^{4} g\right)$ is

Received by the editors January 8, 2006. 
asymptotically flat with zero scalar curvature. One basic fact about this construction is that the blowing-up manifold $\left(S^{3} \backslash\{P\}, G^{4} g\right)$, up to a constant scaling, depends only on the conformal class $[g]$. Precisely, if one replaces $g$ by another metric $\bar{g} \in[g]$ and let $\bar{G}$ be the Green's function associated to $\bar{g}$, then the metric $\bar{G}^{4} \bar{g}$ differs from $G^{4} g$ only by a constant multiple. Therefore, it is of interest to seek conditions on $[g]$ that determine whether $\left(S^{3} \backslash\{P\}, G^{4} g\right)$ has a horizon.

So far, no such a conformal invariant condition has been found. However, there are results where conditions in terms of a single metric are given. In [1], Beig and Ó Murchadha studied the behavior of a critical sequence, i.e. a sequence of metrics $\left\{g_{n}\right\}$ on $S^{3}$ converging to a metric $g_{0}$ with zero scalar curvature. They showed the blowing-up manifold $\left(S^{3} \backslash\{P\}, G_{n}^{4} g_{n}\right)$ has a horizon for sufficiently large $n$. Their idea was further explored by Yan [12]. Given a metric $g$ on $S^{3}$, assuming the diameter of $\left(S^{3}, g\right) \leq D$, the volume of $\left(S^{3}, g\right) \geq V$ and the Ricci curvature of $g$ satisfies $\operatorname{Ric}(g) \geq \mu g$, Yan showed that, for any $r>\frac{3}{2}$, there exists a small positive number $\delta=\delta(\mu, V, D, r) \leq 1$ such that, if $R(g)>0$ and $\|R(g)\|_{L^{r}\left(S^{3}, g\right)}<\delta$, then the blowingup manifold $\left(S^{3} \backslash\{P\}, G^{4} g\right)$ has a horizon.

One question arising from Yan's theorem is whether a positive Ricci curvature metric on $S^{3}$ can produce a blowing-up manifold with a horizon, as it is unclear whether Yan's theorem could be applied when $\mu>0$. Another motivation to this question is, as a positive Ricci curvature metric can be deformed to the standard metric on $S^{3}$ through metrics of positive Ricci curvature, it is of potential interest to study how the horizon disappears in the corresponding deformation of the blowing-up manifold if it exists initially.

In this paper, we focus on conformal classes of metrics with a positive Ricci curvature metric. Our main result is the observation of a volume condition which guarantees non-existence of horizons in the blowing-up manifold. Throughout the paper, $\mathbb{S}^{3}$ denotes $S^{3}$ with the standard metric of constant curvature +1 .

Theorem Let $[g]$ be a conformal class of metrics on $S^{3}$ which has a metric of positive Ricci curvature. Consider

$$
V_{\max }\left(S^{3},[g]\right)=\sup _{\bar{g} \in[g]}\left\{\operatorname{Vol}\left(S^{3}, \bar{g}\right) \mid \operatorname{Ric}(\bar{g}) \geq 2 \bar{g}\right\},
$$

where $\operatorname{Vol}(\cdot)$ is the volume functional. If

$$
V_{\max }\left(S^{3},[g]\right) \geq \frac{1}{2} \operatorname{Vol}\left(\mathbb{S}^{3}\right)
$$

then the blowing-up manifold $\left(S^{3} \backslash\{P\}, G^{4} g\right)$ has no horizon.

We also give an example of $\left(S^{3}, g\right)$ where $\operatorname{Ric}(g)>0$ and $\left(S^{3} \backslash\{P\}, G^{4} g\right)$ does have horizons.

\section{Positive Ricci curvature and maximum volume}

We first explain the volume assumption in the Theorem. Let $M^{n}$ be a smooth, connected, closed manifold of dimension $n \geq 3$. Assume $[g]$ is a conformal class of metrics on $M^{n}$ which has a metric of positive Ricci curvature. One can define

$$
V_{\text {max }}\left(M^{n},[g]\right)=\sup _{\bar{g} \in[g]}\left\{\operatorname{Vol}\left(M^{n}, \bar{g}\right) \mid \operatorname{Ric}(\bar{g}) \geq(n-1) \bar{g}\right\} .
$$


The following result relating $V_{\max }\left(M^{n},[g]\right)$ and the Yamabe constant of $\left(M^{n},[g]\right)$ was observed in [5].

Proposition 1. Let $[g]$ be a conformal class of metrics on $M^{n}$ which has a metric of positive Ricci curvature. Then the Yamabe constant of $\left(M^{n},[g]\right)$ satisfies

$$
Y\left(M^{n},[g]\right) \geq n(n-1) V_{\max }\left(M^{n},[g]\right)^{\frac{2}{n}} .
$$

Proof. By definition,

$$
Y\left(M^{n},[g]\right)=\inf _{v \in W^{1,2}(M)} \frac{\int_{M}\left[c_{n}|\nabla v|_{\bar{g}}^{2}+R(\bar{g}) v^{2}\right] d V_{\bar{g}}}{\left(\int_{M} v^{\frac{2 n}{n-2}} d V_{\bar{g}}\right)^{\frac{n-2}{n}}}
$$

for any $\bar{g} \in[g]$, where $c_{n}=\frac{4(n-1)}{n-2}$.

Assume $\operatorname{Ric}(\bar{g}) \geq(n-1) \bar{g}$, by a result of Ilias [7], which is based on the isoperimetric inequality of Gromov [9], we have

$$
\int_{M}\left[c_{n}|\nabla v|_{\bar{g}}^{2}+n(n-1) v^{2}\right] d V_{\bar{g}} \geq\left(\int_{M} v^{\frac{2 n}{n-2}} d V_{\bar{g}}\right)^{\frac{n-2}{n}} n(n-1) \operatorname{Vol}\left(M^{n}, \bar{g}\right)^{\frac{2}{n}}
$$

for any $v \in W^{1,2}(M)$. Note that $R(\bar{g}) \geq n(n-1)$, hence

$$
\begin{aligned}
Y\left(M^{n},[g]\right) & \geq \inf _{v \in W^{1,2}(M)} \frac{\int_{M}\left[c_{n}|\nabla v|_{\bar{g}}^{2}+n(n-1) v^{2}\right] d V_{\bar{g}}}{\left(\int_{M} v^{\frac{2 n}{n-2}} d V_{\bar{g}}\right)^{\frac{n-2}{n}}} \\
& \geq n(n-1) \operatorname{Vol}\left(M^{n}, \bar{g}\right)^{\frac{2}{n}} .
\end{aligned}
$$

Taking the supremum over $\bar{g} \in[g]$ satisfying $\operatorname{Ric}(\bar{g}) \geq(n-1) \bar{g}$, we have

$$
Y\left(M^{n},[g]\right) \geq n(n-1) V_{\max }\left(M^{n},[g]\right)^{\frac{2}{n}} .
$$

As an immediate corollary, we see the assumption

$$
V_{\max }\left(S^{3},[g]\right) \geq \frac{1}{2} \operatorname{Vol}\left(\mathbb{S}^{3}\right)
$$

in the Theorem implies

$$
\begin{aligned}
Y\left(S^{3},[g]\right) & \geq 6\left(\frac{1}{2}\right)^{\frac{2}{3}} \operatorname{Vol}\left(\mathbb{S}^{3}\right)^{\frac{2}{3}} \\
& =Y\left(R P^{3},\left[g_{0}\right]\right)
\end{aligned}
$$

where $R P^{3}$ is the three dimensional projective space and $g_{0}$ is the standard metric on $R P^{3}$ which has constant sectional curvature +1 . 


\section{An upper bound of the Sobolev constant when a horizon is present}

One basic fact relating the conformal class $[g]$ on $S^{3}$ and the blowing-up metric $h=G^{4} g$ on $\mathbb{R}^{3}=S^{3} \backslash\{P\}$ is

$$
Y\left(S^{3},[g]\right)=8 S(h)
$$

where $S(h)$ is the Sobolev constant of the asymptotically flat manifold $\left(\mathbb{R}^{3}, h\right)[3]$. Recall $S(h)$ is defined by

$$
S(h)=\inf _{u \in W^{1,2}\left(\mathbb{R}^{3}, h\right)}\left\{\frac{\int_{\mathbb{R}^{3}}|\nabla u|_{h}^{2} d V_{h}}{\left(\int_{\mathbb{R}^{3}} u^{6} d V_{h}\right)^{\frac{1}{3}}}\right\} .
$$

The next proposition, which plays a key role in the derivation of the Theorem, was essentially established by Bray and Neves in [3] using the inverse mean curvature flow technique [6]. As the statement of Bray and Neves is different from what we need, we include the proof here.

Proposition 2. Let $h$ be a complete metric on $\mathbb{R}^{3}$ such that $\left(\mathbb{R}^{3}, h\right)$ is asymptotically flat. If $\left(\mathbb{R}^{3}, h\right)$ has nonnegative scalar curvature and has a closed minimal surface, then

$$
S(h)<\frac{1}{8} Y\left(R P^{3},\left[g_{0}\right]\right) .
$$

Proof. Since $\left(\mathbb{R}^{3}, h\right)$ has a closed minimal surface, the outermost minimal surface $\mathcal{S}$ in $\left(\mathbb{R}^{3}, h\right)$, i.e. the closed minimal surface that is not enclosed by any other minimal surface [2], exists and consists of a finite union of disjoint, embedded minimal twospheres and projective planes. As our background manifold is $\mathbb{R}^{3}, \mathcal{S}$ must consist of embedded minimal two-spheres alone, furthermore each component of $\mathcal{S}$ necessarily bounds a three-ball.

We fix a component $\Sigma$ of $\mathcal{S}$ and denote by $\Omega$ the three-ball that $\Sigma$ bounds in $\mathbb{R}^{3}$. Let $\phi$ be the weak solution to the inverse mean curvature flow in $\left(\mathbb{R}^{3} \backslash \bar{\Omega}, h\right)$ with initial condition $\Sigma[6] . \phi$ satisfies

$$
\phi \geq 0,\left.\phi\right|_{\Sigma}=0, \quad \lim _{x \rightarrow \infty} \phi=\infty .
$$

Let $\Sigma_{t}$ be the set $\partial\{u<t\}$ for $t>0$ and $\Sigma_{0}$ be the starting surface $\Sigma$, then the family of surfaces $\left\{\Sigma_{t}\right\}$ satisfies the following properties [6]:

(1) $\left\{\Sigma_{t}\right\}$ consists of $C^{1, \alpha}$ surfaces. For a.e. $t, \Sigma_{t}$ has weak mean curvature $H$ and $H=|\nabla u|_{h}$ for a.e. $x \in \Sigma_{t}$.

(2) $\left|\Sigma_{t}\right|=e^{t}\left|\Sigma_{0}\right|$, where $\left|\Sigma_{t}\right|$ denotes the area of $\Sigma_{t}$.

(3) Since $\left(\mathbb{R}^{3}, h\right)$ has nonnegative scalar curvature, $\Sigma$ is connected and $\mathbb{R}^{3} \backslash \bar{\Omega}$ is simply connected, the Hawking quasi-local mass of $\Sigma_{t}$,

$$
m_{H}\left(\Sigma_{t}\right)=\sqrt{\frac{\left|\Sigma_{t}\right|}{16 \pi}}\left(1-\frac{1}{16 \pi} \int_{\Sigma_{t}} H^{2} d \mu\right),
$$

is monotone increasing. Here $d \mu$ is the induced surface measure.

Now we restrict attention to functions $u \in W^{1,2}\left(\mathbb{R}^{3}, h\right)$ that have the form

$$
u(x)=\left\{\begin{array}{cl}
f(0) & x \in \Omega \\
f(\phi(x)) & x \in \mathbb{R}^{3} \backslash \Omega
\end{array}\right.
$$


for some $C^{1}$ functions $f(t)$ defined on $[0, \infty)$. By the coarea formula and Property 1 above, we have

$$
\begin{aligned}
\int_{\mathbb{R}^{3}}|\nabla u|_{h}^{2} d V_{h} & =\int_{0}^{\infty} f^{\prime}(t)^{2}\left(\int_{\Sigma_{t}} H d \mu\right) d t \\
& \leq \int_{0}^{\infty} f^{\prime}(t)^{2} \sqrt{16 \pi|\Sigma|\left(e^{t}-e^{\frac{t}{2}}\right)} d t
\end{aligned}
$$

where the inequality follows from Property 2, 3 and Hölder's inequality. Similarly, we have

$$
\begin{aligned}
\int_{\mathbb{R}^{3}} u^{6} d V_{h} & \geq \int_{0}^{\infty} f(t)^{6}\left(\int_{\Sigma_{t}} H^{-1} d \mu\right) d t \\
& \geq \int_{0}^{\infty} f(t)^{6} e^{2 t}|\Sigma|^{2}\left[16 \pi|\Sigma|\left(e^{t}-e^{\frac{t}{2}}\right)\right]^{-\frac{1}{2}} d t
\end{aligned}
$$

Therefore,

$$
\frac{\int_{\mathbb{R}^{3}}|\nabla u|_{h}^{2} d V_{h}}{\left(\int_{\mathbb{R}^{3}} u^{6} d V_{h}\right)^{\frac{1}{3}}} \leq \frac{(16 \pi)^{\frac{2}{3}} \int_{0}^{\infty} f^{\prime}(t)^{2}\left(e^{t}-e^{\frac{t}{2}}\right)^{\frac{1}{2}} d t}{\left(\int_{0}^{\infty} f(t)^{6} e^{2 t}\left(e^{t}-e^{\frac{t}{2}}\right)^{-\frac{1}{2}} d t\right)^{\frac{1}{3}}} .
$$

To pick an optimal $f(t)$ that minimizes the right side of (16), we consider the half spatial Schwarzschild manifold

$$
\left(M^{3}, g_{S}\right)=\left(\mathbb{R}^{3} \backslash B_{1}(0),\left(1+\frac{1}{|x|}\right)^{4} \delta_{i j}\right)
$$

and the quotient manifold $\left(\tilde{M}^{3}, \tilde{g}_{S}\right)$ obtained from $\left(M^{3}, g_{S}\right)$ by identifying the antipodal points of $\{|x|=1\}$. Up to scaling, $\left(\tilde{M}^{3}, \tilde{g}_{S}\right)$ is isometric to $\left(R P^{3} \backslash\{Q\}, G_{0}^{4} g_{0}\right)$, the blowing-up manifold of $\left(R P^{3}, g_{0}\right)$ by its Green function at a point $Q$. Hence, the Sobolev constant $S\left(\tilde{g}_{S}\right)$ of $\left(\tilde{M}^{3}, \tilde{g}_{S}\right)$ equals $\frac{1}{8} Y\left(R P^{3},\left[g_{0}\right]\right)$. On the other hand, $S\left(\tilde{g}_{S}\right)$ is achieved by a function $u_{0}$ that is a constant on each coordinate sphere $\{|x|=t\}$ in $\tilde{M}$, and the level set of the solution $\phi_{0}$ to the inverse mean curvature flow starting at $\{|x|=1\}$ in $\left(M, g_{S}\right)$ is also given by coordinate spheres. Therefore, lifted as a function on $\left(M^{3}, g_{S}\right), u_{0}$ has the form of

$$
u_{0}=f_{0} \circ \phi_{0}
$$

for some explicitly determined function $f_{0}(t)$, and

$$
S\left(\tilde{g}_{S}\right)=\frac{\int_{M}\left|\nabla u_{0}\right|_{g_{S}}^{2} d V_{g_{S}}}{\left(\int_{M} u_{0}^{6} d V_{g_{S}}\right)^{\frac{1}{3}}}=\frac{(16 \pi)^{\frac{2}{3}} \int_{0}^{\infty} f_{0}^{\prime}(t)^{2}\left(e^{t}-e^{\frac{t}{2}}\right)^{\frac{1}{2}} d t}{\left(\int_{0}^{\infty} f_{0}(t)^{6} e^{2 t}\left(e^{t}-e^{\frac{t}{2}}\right)^{-\frac{1}{2}} d t\right)^{\frac{1}{3}}},
$$

where the second equality holds because the Hawking quasi-local mass remains unchanged along the level sets of $\phi_{0}$. Now consider $u=f_{0} \circ \phi$ on $\left(\mathbb{R}^{3}, h\right)$. It was verified in [3] that $u \in W^{1,2}\left(\mathbb{R}^{3}, h\right)$. Therefore, we have

$$
\begin{aligned}
S(h) \leq \frac{\int_{\mathbb{R}^{3}}|\nabla u|_{h}^{2} d V_{h}}{\left(\int_{\mathbb{R}^{3}} u^{6} d V_{h}\right)^{\frac{1}{3}}} & \leq \frac{(16 \pi)^{\frac{2}{3}} \int_{0}^{\infty} f_{0}^{\prime}(t)^{2}\left(e^{t}-e^{\frac{t}{2}}\right)^{\frac{1}{2}} d t}{\left(\int_{0}^{\infty} f_{0}(t)^{6} e^{2 t}\left(e^{t}-e^{\frac{t}{2}}\right)^{-\frac{1}{2}} d t\right)^{\frac{1}{3}}} \\
& =S\left(\tilde{g}_{S}\right)=\frac{1}{8} Y\left(R P^{3},\left[g_{0}\right]\right) .
\end{aligned}
$$


To show the strict inequality, we assume $S(h)=\frac{1}{8} Y\left(R P^{3},\left[g_{0}\right]\right)$. Then, $S(h)$ is achieved by $u=f_{0} \circ \phi$. It follows from the Euler-Lagrange equation of the Sobolev functional (11) that $u$ satisfies

$$
\triangle_{h} u+C u^{5}=0 \text { on } \mathbb{R}^{3},
$$

where $C=S(h)\|u\|_{L^{6}\left(\mathbb{R}^{3}, h\right)}^{-4}$. However, $u \equiv f_{0}(0)$ on $\Omega$ and $f_{0}(0) \neq 0$ (Indeed, up to a constant multiple, $\left.f_{0}(t)=\left(2 e^{t}-e^{\frac{t}{2}}\right)^{-\frac{1}{2}}[3]\right)$. Hence, $C=0$, which contradicts to the fact that $u$ is not a constant. Therefore, the strict inequality $S(h)<\frac{1}{8} Y\left(R P^{3},\left[g_{0}\right]\right)$ holds.

Proof of the Theorem: Suppose $\left(S^{3} \backslash\{P\}, G^{4} g\right)$ has a horizon, then it follows from (10) and Proposition 2 that

$$
Y\left(S^{3},[g]\right)<Y\left(R P^{3},\left[g_{0}\right]\right) .
$$

On the other hand, the assumption $V_{\max }\left(S^{3},[g]\right) \geq \frac{1}{2} \operatorname{Vol}\left(\mathbb{S}^{3}\right)$ implies

$$
Y\left(S^{3},[g]\right) \geq Y\left(R P^{3},\left[g_{0}\right]\right)
$$

by (9), which is a contradiction. Hence, there are no horizons in $\left(S^{3} \backslash\{P\}, G^{4} g\right)$.

\section{An example with horizons}

In this section, we provide an example to show that there exist metrics on $S^{3}$ with positive Ricci curvature such that the blowing-up manifolds do have horizons.

Our example comes from a 1-parameter family of left-invariant metrics $\left\{g_{\epsilon}\right\}$ on $S^{3}$, commonly known as the Berger metrics. Precisely, we think $S^{3}$ as the Lie Group

$$
S U(2)=\left\{\left(\begin{array}{cc}
z & -w \\
\bar{w} & \bar{z}
\end{array}\right):|z|^{2}+|w|^{2}=1\right\},
$$

where the Lie algebra of $S U(2)$ is spanned by

$$
X_{1}=\left(\begin{array}{cc}
i & 0 \\
0 & -i
\end{array}\right), X_{2}=\left(\begin{array}{cc}
0 & 1 \\
-1 & 0
\end{array}\right) \text {, and } X_{3}=\left(\begin{array}{cc}
0 & i \\
i & 0
\end{array}\right) \text {. }
$$

Then $\left\{g_{\epsilon}\right\}$ is defined by declaring $X_{1}, X_{2}, X_{3}$ to be orthogonal, $X_{1}$ to have length $\epsilon$ and $X_{2}, X_{3}$ to be unit vectors. Note that scalar multiplication on $S^{3} \subset \mathbb{C}^{2}$ corresponds to multiplication on the left by matrices $\left(\begin{array}{cc}e^{i \theta} & 0 \\ 0 & e^{-i \theta}\end{array}\right)$ on $S U(2)$, hence $X_{1}$ is exactly tangent to the circle fiber of the Hopf fibration

$$
\pi: S^{3} \longrightarrow S^{2}=S^{3} / S^{1}
$$

and $g_{\epsilon}$ shrinks the circle fiber as $\epsilon \rightarrow 0$. One fact of $g_{\epsilon}$ for small $\epsilon$ is that all sectional curvature of $\left(S^{3}, g_{\epsilon}\right)$ lies in the interval $\left[\epsilon^{2}, 4-3 \epsilon^{2}\right]$ (see [10]), in particular $g_{\epsilon}$ has positive Ricci curvature.

Proposition 3. Let $P \in S^{3}$ be a fixed point and $G_{\epsilon}$ be the Green's function of the conformal Laplacian of $g_{\epsilon}$ at $P$. Then $\left(S^{3} \backslash\{P\}, G_{\epsilon}^{4} g_{\epsilon}\right)$ has a horizon for $\epsilon$ sufficiently small. 
Proof. For each $\epsilon \in(0,1]$, we consider the rescaled metric $\bar{g}_{\epsilon}=\epsilon^{-2} g_{\epsilon}$ and the Green's function $\bar{G}_{\epsilon}$ associated to $\bar{g}_{\epsilon}$ at $P$. Then, with respect to $\bar{g}_{\epsilon}, X_{1}$ becomes a unit vector and $X_{2}, X_{3}$ have large length $\epsilon^{-1}$ as $\epsilon \rightarrow 0$. Let $U \subset S^{3}$ be a fixed neighborhood of $P$ such that $\left.\pi\right|_{U}$ is a trivial fiberation. Let $O$ be a fixed point in the product manifold $S^{1} \times \mathbb{R}^{2}$. By a scaling argument, there exists a family of diffeomorphisms

$$
\Psi_{\epsilon}: U \longrightarrow \Psi_{\epsilon}(U) \subset S^{1} \times \mathbb{R}^{2},
$$

such that $\Psi_{\epsilon}(P)=O \in \Psi_{\epsilon}(U),\left\{\Psi_{\epsilon}(U)\right\}_{1 \geq \epsilon>0}$ forms an exhaustion family of $S^{1} \times \mathbb{R}^{2}$ as $\epsilon \rightarrow 0$, and the push forward metrics $\hat{g}_{\epsilon}=\Psi_{\epsilon}^{-1 *}\left(\left.\bar{g}_{\epsilon}\right|_{U}\right)$ on $\Psi_{\epsilon}(U)$ converge in $C^{2}$ norm on compact sets to a flat metric $\hat{g}$ on $S^{1} \times \mathbb{R}^{2}$. Now fix another point $Q \in \Psi_{1}(U)$ that is different from $O$ and consider the normalized function

$$
\hat{G}_{\epsilon}(x)=\frac{\bar{G}_{\epsilon} \circ \Psi_{\epsilon}^{-1}(x)}{\bar{G}_{\epsilon} \circ \Psi_{\epsilon}^{-1}(Q)}
$$

for $x \in \Psi_{\epsilon}(U) \backslash\{O\}$. Then $\hat{G}_{\epsilon}$ satisfies

$$
\left\{\begin{array}{rl}
8 \triangle_{\hat{g}_{\epsilon}} \hat{G}_{\epsilon}-R\left(\hat{g}_{\epsilon}\right) \hat{G}_{\epsilon} & =0 \text { on } \Psi_{\epsilon}(U) \backslash\{O\} . \\
\hat{G}_{\epsilon} & =1 \text { at } Q
\end{array} .\right.
$$

Since $\hat{G}_{\epsilon}$ is positive and $\hat{g}_{\epsilon}$ converges to $\hat{g}$ as $\epsilon \rightarrow 0$, it follows from the Harnack inequality that $\hat{G}_{\epsilon}$ coverges to a positive function $\hat{G}$ on $\left(S^{1} \times \mathbb{R}^{2}\right) \backslash\{O\}$ in $C^{2}$ norm on any compact set away from $\{O\}$. Furthermore, $\hat{G}$ satisfies

$$
\left\{\begin{array}{rl}
\triangle_{\hat{g}} \hat{G} & =0 \text { on }\left(S^{1} \times \mathbb{R}^{2}\right) \backslash\{O\} \\
\hat{G} & =1 \text { at } Q
\end{array} .\right.
$$

On the other hand, the fact that the geodesic ball in $\left(S^{1} \times \mathbb{R}^{2}, \hat{g}\right)$ only has quadratic volume growth implies $\left(S^{1} \times \mathbb{R}^{2}, \hat{g}\right)$ does not have a positive Green's function for the usual Laplacian $\triangle_{\hat{g}}[4]$. Therefore, $\hat{G} \equiv 1$ on $\left(S^{1} \times \mathbb{R}^{2}\right) \backslash\{O\}$. Hence, the metrics $\hat{G}_{\epsilon}^{4} \hat{g}_{\epsilon}$ converge to $\hat{g}$ in $C^{2}$ norm on any compact set away from $\{O\}$. Now let $V \subset S^{1} \times \mathbb{R}^{2}$ be a small open ball containing $O$ such that $\partial V$ is an embedded two sphere whose mean curvature vector computed with respect to $\hat{g}$ points towards $O$. Then, for sufficiently small $\epsilon$, the mean curvature vector of $\partial V$ computed with respect to $\hat{G}_{\epsilon}^{4} \hat{g}_{\epsilon}$ still points towards $O$. As $\left(\Psi_{\epsilon}(U), \hat{G}_{\epsilon}^{4} \hat{g}_{\epsilon}\right)$ is isometric to $\left(U, \bar{G}_{\epsilon}^{4} \bar{g}_{\epsilon}\right)$, the mean curvature vector of the boundary of $\Psi_{\epsilon}^{-1}(V)$ in $\left(S^{3} \backslash\{P\}, \bar{G}_{\epsilon}^{4} \bar{g}_{\epsilon}\right)$ must point towards the blowing-up point $P$. On the other hand, as $\left(S^{3} \backslash\{P\}, \bar{G}_{\epsilon}^{4} \bar{g}_{\epsilon}\right)$ is asymptotically flat, its infinity is foliated by two spheres whose mean curvature vector points away from $P$. Therefore, it follows from standard geometric measure theory that there exists an embedded minimal two sphere in $\Psi_{\epsilon}(V)$, hence $\left(S^{3} \backslash\{P\}, \bar{G}_{\epsilon}^{4} \bar{g}\right)$ has a horizon.

\section{Acknowledgments}

I want to thank Justin Corvino and Rick Schoen for helpful discussions. 


\section{References}

[1] R. Beig and N. Ó Murchadha, Trapped surfaces due to concentration of gravitational radiation, Phys. Rev. Lett. 66 (1991), no. 19, 2421-2424.

[2] H. L. Bray, Proof of the Riemannian Penrose inequality using the positive mass theorem, J. Differential Geom. 59 (2001), no. 2, 177-267.

[3] H. L. Bray and A. Neves, Classification of prime 3-manifolds with Yamabe invariant greater than $\mathbb{R P}^{3}$, Ann. of Math. (2) 159 (2004), no. 1, 407-424.

[4] S. Y. Cheng and S. T. Yau, Differential equations on Riemannian manifolds and their geometric applications, Comm. Pure Appl. Math. 28 (1975), no. 3, 333-354.

[5] P. Guan and G. Wang, Conformal deformations of the smallest eigenvalue of the Ricci tensor, Max Planck Institute Preprint Nr. 43/2005 (2005)

[6] G. Huisken and T. Ilmanen, The inverse mean curvature flow and the Riemannian Penrose inequality, J. Differential Geom. 59 (2001), no. 3, 353-437.

[7] S. Ilias, Constantes explicites pour les inégalités de Sobolev sur les variétés riemanniennes compactes, Ann. Inst. Fourier (Grenoble) 33 (1983), no. 2, 151-165.

[8] W. Meeks, III, L. Simon, and S. T. Yau, Embedded minimal surfaces, exotic spheres, and manifolds with positive Ricci curvature, Ann. of Math. (2) 116 (1982), no. 3, 621-659.

[9] V. D. Milman and G. Schechtman, Asymptotic theory of finite-dimensional normed spaces, Vol. 1200 of Lecture Notes in Mathematics, Springer-Verlag, Berlin (1986), ISBN 3-540-16769-2. With an appendix by M. Gromov.

[10] P. Petersen, Riemannian geometry, Vol. 171 of Graduate Texts in Mathematics, Springer-Verlag, New York (1998), ISBN 0-387-98212-4.

[11] R. Schoen and S. T. Yau, The existence of a black hole due to condensation of matter, Comm. Math. Phys. 90 (1983), no. 4, 575-579.

[12] Y. Yan, The existence of horizons in an asymptotically flat 3-manifold, Math. Res. Lett. 12 (2005), no. 2-3, 219-230.

School of Mathematical Sciences, Monash University, Vic 3800, Australia

E-mail address: Pengzi.Miao@sci.monash.edu.au 